\title{
Unsupervised Discovery of Phonological Categories through Supervised Learning of Morphological Rules
}

\author{
Walter Daelemans* \\ CL \& AI, Tilburg University \\ P.().Box 90153, 5000 L. Tilburg \\ The Netherlands \\ walter.daelemans@kub.n1
}

\begin{abstract}
We describe a case study in the application of symbolic machine learning technicues for the discovery of linguistic rules and categories. $\Lambda$ supervised rule induction algorithm is used to learn to predict the correct diminutive suffix given the phonological representation of Dutch nouns. The system produces rules which are comparable to rules proposed by linguists. Furthermore, in the process of learning this morphological task, the phonemes used are grouped into phonologically relevant, catcgories. Wo discuss the rolevance of our method for linguistics and language tochnology.
\end{abstract}

\section{Introduction}

This paper shows how machine learning techniques can be used to induce linguistically relevant rules and categories from data. Statistical, connectionist, and machine learning induction (datiaoriented approaches) are currently used mainly in language engincering applications in order to alleviate the linguistic knowledge acquisition bottleneck (the fact that lexical and grammatical knowledge usually has to be reformulated from scratch whenever a new application has to be built or an existing application ported to a new domain), and to solve problems with robustness and coverage inherent in knowledge-based (theory-oriented, hand-crafting) approaches. Iinguistic rolevance or inspectability of the induced knowledge is nsually not an issue in this type of research. In linguistics, on the other hand, it is usually agreed that while computer modeling is a uscful (or cssential) tool for cuforcing internal consistency, completeness, and empirical validity of the linguistic theory boing modeled, its role in formulating or evaluating linguistic theories is minimal.

In this paper, we argue that machine learning techniques can also assist in linguistic theory for-

\footnotetext{
${ }^{*}$ Visiting fellow at NIAS (Netherlands Institute for Advanced Studies), Wassenaar, The Netherlands.
}

\author{
Peter Berck and Steven Gillis \\ Iinguistics, University of Antwerp \\ Universiteitsplein 1, 2610 Wilrijk \\ Belgium \\ steven.gillis@uia.ua.ac.be \\ peter berck@uia.ua.ac. be
}

mation by providing a new tool for the evaluattion of linguistic hypotheses, for the extraction of rules from corporal, and for the discovery of useful linguistic categories. As a case study, we apply Quinlan's C4.5 inductive machine learning method (Quinlan, 1993) to a particular linguistic task (diminutive formation in Dutch) and show that it can bo used (i) to test linguistic hypotheses about this process, (ii) to discover interesting morphological rules, and (iii) discover interesting phonological categories. Nothing hinges on our choice of (4.5 as a rule induction mechanism. Wo chose it because it is an easily available and sophisticated instance of the class of rule induction algorithrus.

$\Lambda$ second focus of this paper is the interaction between supervised and unsupervised machine loarning methods in linguistic discovery. In supervised learning, tho learner is prosented a set, of examples (the experience of the system). These examples consist of an input output association (in our case, e.g., a representation of a noun as input, and the corresponding diminutive suffix as output). Unsupervised learning methods do not provide the learner with information about the output to be generated; only the inputs are presented to the learner as experience, not the target outputs.

Unsupervised learning is necessarily more limited than supervised learning; the only information it has to construct categories is the similarity between inputs. Unsupervised learning has been successfully applied e.g. for the discovery of syntactic categorios from corpora on the basis of distributional information about words (Finch and Chater 1992, Hughes 1994, Schütze 1995). Wo will show that it is possible and useful to make use of unsupervised learning relative to a particular task which is being learned in a supervised way. In our experiment, phonological categories are discovered in an unsupervised way, as a side-effect of the supervised leaning of a morphological problem. We will also show that this raises interesting questions about the task-dependence of linguistic category systoms. 


\section{Supervised Rule Induction with C4.5}

For the experiments, we used C4.5 (Quinlan, 1993). Although several decision tree and rule induction variants have been proposed, we chose this program because it is widely available and reasonably well tested. C4.5 is a TDIDT (Top Down Induction of Decision Trees) decision tree learning algorithm which constructs a decision tree on the basis of a set of examples (the training set). This decision tree has tests (feature names) as nodes, and feature values as branches between nodes. The leaf nodes are labeled with a category name and constitute the output of the system. A decision tree constructed on the basis of examples is used after training to assign a class to patterns. To test whether the tree has actually learned the problem, and has not just momorized the items it was trained on, the generalization accuracy is measured by testing the learned tree on a part of the dataset not used in training.

The algorithm for the construction of a C4.5 decision tree can be easily stated. Given are a training set $T$ (a collection of examples), and a finite number of classes $C_{1} \ldots C_{n}$.

1. If $T$ contains one or more cases all belonging to the same class $C_{j}$, then the decision treo for $T$ is a leaf node with category $C_{j}$.

2. If $T$ is empty, a category has to be found on the basis of other information (e.g. domain knowledge). The heuristic used here is that the most frequent class in the initial training set is used.

3. If $T$ contains different classes then

(a) Choose a test (fcature) with a finite number of outcomes (values), and partition $T$ into subsets of examples that have the same outcome for the test chosen. The decision tree consists of a root node containing the test, and a branch for each outcome, each branch leading to a subset of the original set.

(b) Apply the procedure recursively to subsets created this way.

In this algorithm, it is not specificd which test to choose to split a node into subtrees at some point. Taking one at random will usually result in large decision trees with poor generalization performance, as uninformative tests may be chosen. Considering all possible trees consistent with the data is computationally intractable, so a reliable heuristic test selection method has to be found. The method used in C4.5 is based on the concept of mutual information (or information gain). Whenever a test has to be selected, the feature is chosen with the highest information gain. This is the feature that reduces the information entropy of the training (sub) sct on average most, when its value would be known. For the computation of information gain, see Quinlan (1993).

Decision trees can be easily and automatically transformed into scts of if-then rules (production rules), which are in general easier to understand by domain experts (linguists in our case). In C4.5 this tree-to-rule transformation involves additional statistical evaluation resulting sometimes in a rule set more understandable and accurate than the corresponding decision tree.

The C4.5 algorithm also contains a value grouping mothod which, on the basis of statistical information, collapses different values for a feature into the same catcgory. 'That way, more concise decision trees and rules can be produced (instead of several different branches or rule conditions for each value, only one branch or condition has to bo defined, making reference to a class of values). The algorithm works as a heuristic scarch of the search space of all possible partitionings of the values of a particular feature into sets, with the formation of homogeneous nodes (nodes representing examples with predominantly the same category) as a heuristic guide. See Quinlan (1993) for more information.

\section{Diminutive Formation in Dutch}

In the remainder of this paper, we will describe a case study of using C4.5 to test linguistic hypotheses and to discover regularities and categories. The case study concerns allomorphy in Dutch diminutive formation, "one of the more vexed problems of Dutch phonology (...) [and] one of the most spectacular phenomena of modcrn Dutch morphophonemics" (Trommelen 1983). Diminutive formation is a productive morphological rule in Dutch. Diminutives are formed by attaching a form of the Germanic: suffix -tje to the singular base form of a noun. The suffix shows allomorphic variation (Table 1 ).

\begin{tabular}{l|l|l} 
Noun & Form & Suffix \\
\hline huis (housc) & huisje & $-j e$ \\
man (man) & mannetjo & $-e t j e$ \\
raam (window) & raampjo & $-p j e$ \\
woning (house) & woninkjo & $-k j e$ \\
baan (job) & baantje & $-t j e$
\end{tabular}

Table 1: Allomorphic variation in Dutch diminutives.

The frequency distribution of the different categories is given in Table 2. Wo distinguish between database frequency (frequency of a suffix in a list of 3900 diminutive forms of nouns we took from the CELFX lexical database ${ }^{1}$ ) and corpus

\footnotetext{
${ }^{1}$ Developed by the Center for Lexical Information, Nijmegen. Distributed by the Linguistic Data Consortium
} 
frequency (froquency of a suffix in the text corpus on which the word list was based).

\begin{tabular}{|l|r|r|r|}
\hline Suflix & Frequency & Database \% & Corpus \\
\hline tjo & 1897 & $48.7 \%$ & $50.9 \%$ \\
jo & 1462 & $37.5 \%$ & $30.4 \%$ \\
etjo & 357 & $9.7 \%$ & $10.9 \%$ \\
pje & 104 & $2.7 \%$ & $4.0 \%$ \\
kje & 77 & $2.0 \%$ & $3.8 \%$ \\
\hline
\end{tabular}

Thable 2: lexicon and corpus frecunency of allomorphis.

Iistorically, different analyses of diminutive formation have taken a different view of the rules that govern the choice of the diminutive suflix, and of the linguistic concents playing a role in these rules (see o.g. Te. Winkel 1866, Krnizinga 1915, Cohen 1958, and references in Thommelon 1983). In the latter, it is argued that diminutive formation is a local process, in which concents such ats word stress and morphological structure (proposed in the earlier analyses) do not play a role. 'The rhyme of the last syllable of the nom is necessary and sulliciont to prodict the correct allomorph. 'The natural categories (or features) which are hypothesised in her rules inchudo obstrueents, sonorants, and the class of bimoraic vowels (consisting of long vowels, diphtongs and sehwa).

Dimimtive formation is a small linguistic: domain for which different competing theories have been proposed, and for which different generalizattions (in terms of rules and linguistic: catcegories) have becn proposed. What we will show next is how machine learning technigues may be nsed to (i) test competing hypotheses, (ii) discover generalizations in the data which can then be compared to the generalizations formulated by linguists, and (iii) discover phonological catcgories in an unsupervised way by supervised learning of climinutive suffix prediction.

\section{Experiments}

For each of the 3900 nouns we collected, the following information was kept.

1. The phoneme transcription describing the syllable structure (in terms of onsct, nucleus, and coda) of the last three syllables of the word. Missing slots are inclicated with $=$.

2. For each of these three last syllables the presence or absence of stress.

3. The corresponding diminutive allomorph, abbreviated to $\mathrm{F}$ (-etje), I' (-t.je), of (-je), K (kje), and $\mathrm{I}^{3}(-\mathrm{p} j \mathrm{je})$. 'This is the 'catcgory' of the word to be learmed by the learner.

Some examples are given below (the word itsolf and its gloss are provided for convenience and were not used in the experiments).

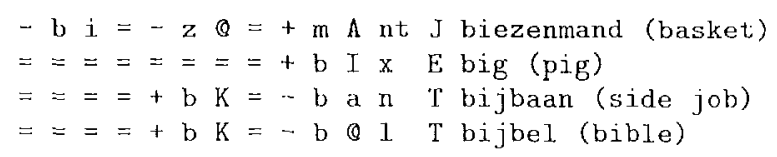

\subsection{Fxperimental Method}

The experimential set-np used in all experiments consisted of a ten-fold coss-validation oxperiment (Weiss \& Kulikowski 1991). In this sct-11p, the datiabase is partitionod ten times, aach with a dilferent $10 \%$ of the datiaset as the test part, and the? remaining $90 \%$ as training part. For each of the ton simmlations in our experinents, the test part was used to test generalization performance. The success rate of an algorithm is obtained by calculating the average acemalcy (number of test, pat, tern categories correctly predicted) over the ten test sets in the ten-fold cross-validation experiinent.

\subsection{Learnability}

The experiments show that the diminutive formation problen is learnable in a clata-oriented way (i.e. by extraction of regularitios from exanples, withont any a priori knowledge abont the domain ${ }^{2}$ ). The average accuracy on musecen test datia of $98.4 \%$ shomkl be compared to baseline performance measures based on probabilitybased gruessing. This baseline woukd be an alecat racy of about $40 \%$ for this problem. This shows that the problem is almost perfoctly learnable by induction. It shoukd be noted that CtidiX contains a number of coding crrors, so that some of the 'wrong' allomorphs predicted by the machine: learning system were actually correct, we clid not, correct for this.

In the next three sections, we will describe the resultis of the experiments; first on the task of conparing conflicting theoretical hypotheses, then on discovering linguistic genemalizations, and finally on unsupervised discovery of phonological categorics.

\section{$5 \quad$ Linguistic Hypothesis Testing}

(On the basis of the analysis of Dutch climinutive formation by Trommelen (1983), discussed briefly in Section 3, the following hypotheses (among oth(rs) can be formulated.

1. Only information about the last syllable is relevant in predicting the correct allomorph.

2. Information about the onsed of the last syllable is irrelevant in predicting the correct alkomoryh.

3. Stress is irrelevant, in predicting the correct allomorph.

\footnotetext{
${ }^{2}$ Lexcept; syllable structure.
} 
In other words, information about the rhyme of the last syllable of a noun is necessary and sufficient to predict the correct allomorph of the diminutive suffix. To test these hypotheses, wo performed four experiments, training and testing the C4.5 machine learning algorithm with four different corpora. These corpora contained the following information.

1. All information (stress, onset, nucleus, coda) about the three last syllables (3-SYLL corpus).

2. All information about the last syllable (SONC corpus).

3. Information about the last syllable without stress (ONC corpus).

4. Information about the last syllable without stress and onset ( $\mathrm{NC}$ corpus)

\subsection{Results}

Table 3 lists the learnability results. The generalization error is given for each allomorph for the four different training corpora.

\begin{tabular}{|l||rr|rr|rr|rr|}
\hline \multicolumn{1}{|c||}{} & \multicolumn{8}{c|}{ Errors and Error percentages } \\
\hline \hline Suffix & 3 sYLL & \multicolumn{2}{|c|}{ SONC } & \multicolumn{2}{c|}{ ONC } & \multicolumn{2}{|c|}{ NC } \\
\hline Total & 61 & 1.6 & 79 & 2.0 & 80 & 2.0 & 77 & 2.0 \\
\hline -tje & 13 & 0.7 & 13 & 0.7 & 14 & 0.7 & 14 & 0.7 \\
-je & 16 & 1.1 & 15 & 1.0 & 16 & 1.1 & 14 & 1.0 \\
- etje & 26 & 7.3 & 49 & 13.7 & 48 & 13.5 & 44 & 12.3 \\
-kje & 4 & 5.2 & 0 & 0 & 0 & 0 & 0 & 0 \\
- pje & 2 & 1.9 & 2 & 1.9 & 2 & 1.9 & 5 & 4.8 \\
\hline
\end{tabular}

Table 3: Error of C4.5 on the different corpora.

The overall best results are achieved with the most elaborate corpus (containing all information about the three last syllables), suggesting that, contra Trommelen, important information is lost by restricting attention to only the last syllable. As far as the different encodings of the last syllable are concerned, however, the learnability cxperiment coroborates Trommelen's claim that stress and onset are not necessary to predict the correct diminutive allomorph. When we look at the error rates for individual allomorphs, a more complex picture emerges. 'The crror rate on -etje dramatically increases (from $7 \%$ to $14 \%$ ) when restricting information to the last syllable. The -kje allomorph, on the other hand, is learned perfectly on the basis of the last syllablc alone. What has happened here is that the learning method has overgeneralized a rule predicting -kje after tho velar nasal, because the data do not contain enough information to correctly handle the notoriously difficult opposition between words like leerling (pupil, takes - etje) and koning (king, takes -kje). Furthermore, the error rate on $-p j e$ is doubled when onset information is left out from the corpus.
We can conclude from these experimonts that although the broad lines of the analysis by Trommelen (1983) are correct, the learnability results point at a number of problems with it (notably with -kje versus - etje and with - pje). We will move now to the use of inductive learning algorithms as a generator of generalizations about the domain, and compare these generalizations to the analysis of Trommelen.

\section{Supervised Learning of Linguistic Generalizations}

When looking only at the rhyme of the last syllable (the NC corpus), the decision tree generated by $\mathrm{C} 4.5$ looks as follows:

Decision Tree:

coda in $\{r k, n t, l t, r t, p, k, t, s t, s, t s, r s, r p, f$, $\mathrm{x}, 1 \mathrm{k}, \mathrm{Nk}, \mathrm{mp}, \mathrm{xt}, \mathrm{rst}, \mathrm{ns}, \mathrm{nst}$, $\mathrm{rx}, \mathrm{kt}, \mathrm{ft}, \mathrm{lf}, \mathrm{mt}, \mathrm{lp}, \mathrm{ks}, \mathrm{ls}, \mathrm{kst}, \mathrm{Ix}\}: \mathrm{J}$

coda in $\{n,=, 1, j, r, m, N, r n, r m, w, 1 m\}$ :

I nucleus in $\{I, A\}, 0, E$,$\} :$

I I coda in $\{n, 1, r, m\}: E$

I coda in $\{=, j, r n\}: T$

1 coda in $\{\mathrm{rm}, \mathrm{Im}\}: \mathrm{p}$

I $\operatorname{cod} a=N$ :

| 1 nucleus $=\mathrm{I}: \mathrm{K}$

I I nucleus in $\{A, 0, E\}: E$

nucleus in $\{K, a, e, u, M, 0, y, o, i, L), I,,<\}$ :

I coda in $\{n,=, 1, j, r, r n, w\}: T$

I $\operatorname{coda}=\mathrm{m}: \mathrm{P}$

Notice that the phoneme representation used by CELEX (called DISC) is shown here instead of the more standard IPA font, and that the value grouping mechanism of $\mathrm{C} 4.5$ has created a number of phonological categories by collapsing different phonemes into sets indicated by curly brackets.

This decision tree should be read as follows: first check the coda (of the last syllable). If it ends in an obstruent, the allomorph is -je. If not, check the nucleus. If it is bimoraic, and the coda is $/ \mathrm{m} /$, decide - pje, if the coda is not $/ \mathrm{m} /$, decide -tje. When the coda is not an obstruent, the nucleus is short and the coda is /ng/, we have to look at the nucleus again to decide betwcen -kje and-etje (this is where the overgeneralization to $-k j e$ for words in -ing occurs). Finally, the coda (nasa-liquid or not) helps us distinguish between -etje and -pje for those cases where the nucleus is short. It should be clear that this tree can casily be formulated as a set of rules without loss of accuracy.

An interesting problem is that the -etje versus -kje problem for words ending in -ing could hot be solved by referring only to the last syllable (C4.5 and any other statistically based induction algorithm overgeneralize to $-k j e$ ). The following is the knowledge derived by $\mathrm{C} 4.5$ from the full corpus, with all information about the three last syllables (the 3 SYLL corpus). We provide the rule version of the inferred knowledge this time. 


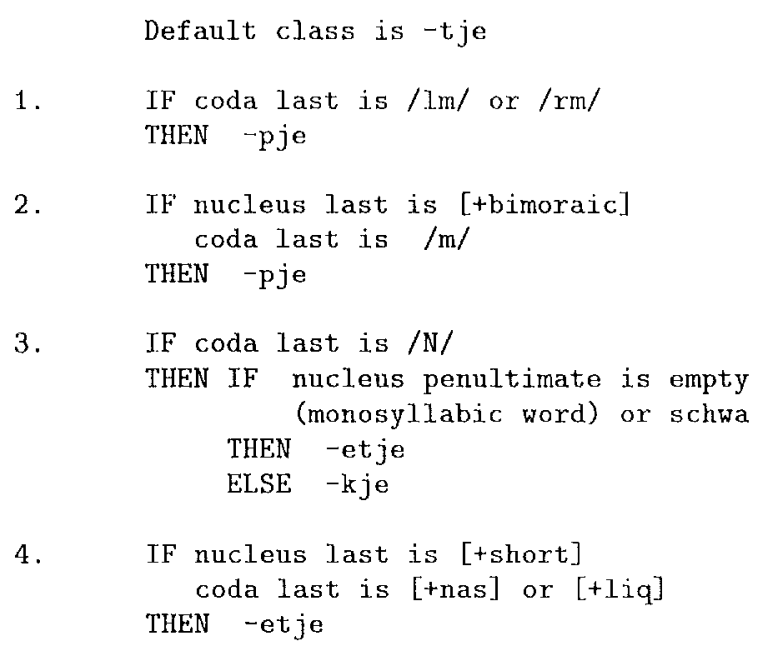

5. IF coda last is [tobstruent] THEN -je

The default class is -tje, which is the allomorph chosen when none of the other rules apply. 'This explains why this rule set looks simpler than the decision tree earlier.

The first thing which is interesting in this rule set, is that only three of the twelve presented features (coda and nucleus of the last syllable, nucleus of the ponultimate syllable) are used in tho rules. Contrary to the hypothesis of Trommelen, apart from the rhyme of the last syllable, the n11cleus of the penultimate syllable is taken to bo relevant, as well.

The induced rules roughly correspond to the previous decision tree, but in addition a solution is provided to the -etje versus - kje problem for words ending in -ing (rule 3) making use of information about the nucleus of the penultimate syllable. Rule 3 states that words ending in /ng/ get -etje as diminutive allomorph when they are monosyllables (uucleus of the penultimate syllable is empty) or when they have a schwa as penultimate nuclens, and -kije otherwise. As far as wo now, this gencralization has not been proposed in this form in the published literature on diminutive formation.

We conclude from this part of the experiment that the machine learning method has succeeded in extracting a sophisticated set of linguistic rules from the examples in a purcly clata-oricnted way, and that these rules aro formulated at a levol that makes their use in the development of linguistic: theories possible.

\section{Discovery of Phonological Categories}

Tho structure the phoneme inventory of a language, linguists define foatures. 'These can be interpreted as sets of speech sounds (categories): e.g. the category (or feature) labial groups those spech somends that involve the lips as an active artic- wator. Speech sounds bolong to different categories, i.e., are clefined by different features. E.g. $b$ is voiceless, a coronal, and a stop). Categorios proposed in phonology are inspired by articulatory, acoustic or perceptual phonetic differences between speech sounds. They are also proposed to allow an optimally conciso or elegant formulation of rules for the description of phonological or morphological processes. E.g., the so-called major class features (obstruents, nasals, liquids, glides, vowels) efficiently explain syllable structure computation, but are of little use in the definition of rules describing assimilation. For assimilation, place of articulation foatures are best nsed. This situation has led to the proposal of nany different phonological category systems.

While constructing the decision tree (see previous section), several phonologically rolevant categories are 'discovered' by the value grouping' mechanism in C4.5, inchuding the nasals, the licnids, the obstruents, the short vowels, and the bimoraic vowels. This last category corresponds completely with the (then now) category hypothesised by 'Trommelen and containing the long vowols, the diphtongs and the schwa. In other words, the learning algorithm has discovered this set of phomemes to be a useful category in solving the climimutive formation problem by providing an oxlensional definition of it (a list of the instances of the category).

This raises the question of the task-dependence of linguistic categories. Similar experiments in Dutch plural formation, for example, fail to produce the category of bimoraic vowels, and for some tasks, categories show up which have no ontological status in linguistics. In other words, making category formation dependent on the task to be learned, undermines the traditional linguistic; ideas about, absolute, task-independent (and even langlage-indepondent) categories. Wo present, here a new methodology with which this funclamental issue in linguistics can be investigated: catogory systoms extracted for difforent tasks in different languages can be studied to see which categories (if any) truely have a universal status. This is subject, for further resoarch. It would also be useful to study the induced categories when intensional descriptions (feature representations) are used as input instead of extensional (escriptions (phonernes).

We also experimented with a simpler alternative to the computationally complex heuristic category formation algorithm used by C4.5. 'This method is inspired by machine learning work on value difference metrics (Stanfill \& Waltz, 1986; Cost \& Salyberg, 1993). Starting from the training set of the supervised learning experiment (the set of input output mappings used by the system to extract rules), we select a particular feature (c.g. the cola of the last syllable), and compute a table as- 
sociating with each possible value of the fcature the number of times the pattern in which it occurs was assigned to cach different catcgory (in this case, each of the the five allomorphs). This produces a table with for each value a distribution over categories. This table is then used in standard clustering approaches to derive categories of values (in this case consonants). The following is one of these clustering results. The example shows that this computationally simple approach also succeeds in discovering categories in an unsupervised way on the basis of data for supervised lcarning.

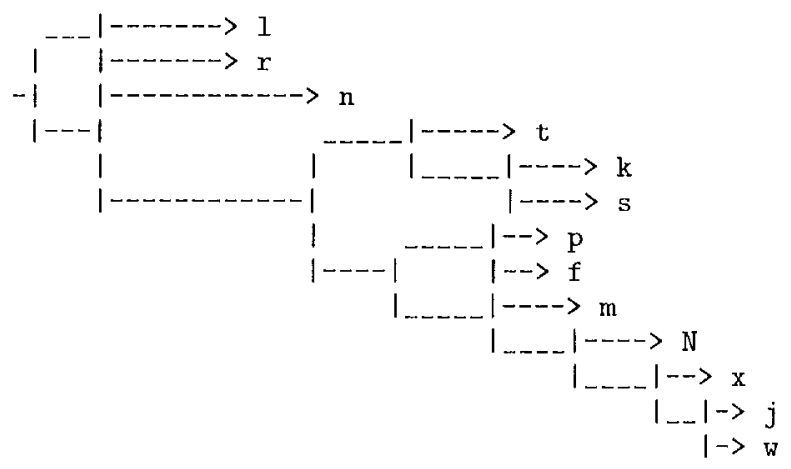

Several categories, relevant for diminutive formation, such as liquids, nasals, the velar nasal, semi-vowels, fricatives etc., are reflected in this hicrarchical clustering.

\section{Conclusion}

We have shown by example that machine learning techniques can profitably be used in linguistics as a tool for the comparison of linguistic theories and hypotheses or for the discovery of new linguistic theories in the form of linguistic rules or catcgories.

The case study we presented concerns diminutive formation in Dutch, for which we showed that (i) machine learning techniques can be used to corroborate and falsify some of the existing theories about the phenomenon, and (ii) machine learning techniques can be used to (re)discover interesting linguistic rules (c.g. the rule solving the -etje versus -kje problem) and categories (e.g. the category of bimoraic vowels).

The extracted system can of course also be used in language technology as a data-oriented system for solving particular linguistic tasks (in this case diminutive formation). In order to test the usability of the approach for this application, we compared the performance of the extracted rule system to the performance of the hand-crafted rule system proposed by Trommelen. Table 4 shows for each allomorph the number of crrors by the C4.5 rules (trained using corpus $\mathrm{NC}$, i.e. only the rhyme of the last syllable) as opposed to an implcmentation of the rules suggested by Trommelen. One problem with the latter is that they often suggest more than one allomorph (the rules are not mutually exclusive). In those cases where more than one rule applies, a choice was made at random.

\begin{tabular}{|l|r|r|}
\hline Suffix & Trommelen & C4.5 \\
\hline -tje & 53 & 11 \\
-je & 12 & 12 \\
-etje & 28 & 39 \\
-kije & 38 & 0 \\
-pje & 21 & 4 \\
\hline Total & 152 & 66 \\
\hline
\end{tabular}

Table 4: Comparison of accuracy between handcrafted and induced rules.

The comparison shows that C4.5 did a good job of finding an elegant and accurate rule-based description of the problem. This rule set is useful both in linguistics (for cvaluation, refinemont, and discovery of theories) and in language technology.

\section{References}

Cohen, A. Het Nederlandse diminuticfsuffix; een morfologische proeve. De Nicuwe Taalgids, 51, 40-45, 1958.

Cost, S. and Salzberg, S. 'A weighted nearest. neighbor algorithm for lcarning with symbolic, features.' Machine Learning, 10, 57 78, 1993.

Finch, S. \& N. Chater. 'Bootstrapping Syntactic Categories Using Statistical Mcthods', in: W.Daelemans \& D.Powers (eds.), Background and Lxperiments in Machine Learning of Natural Lanquage, Tilburg University, ITK, 1992.

Hughes, J. 'Automatically Acquiring a Classification of Words', PhD dissertation, University of Leeds, School of Computer Studies, 1994.

Kruisinga, F. De vorm van verkleinwoorden. De Nieuwe Taalgids, 9, 96-97, 1915.

Quinlan, J. R. C4.5 Programs for machine learning 1993.

Schütze, H., Ambiguity in Language Learning: Computational and Cognitive Models, PhD dissertation, Stanford University, Department of Linguistics, 1995.

Stanfill, C. and Waltz, D.Is. 'Toward Memorybascd Reasoning'. Communications of the ACM 29, 1986, 1213-1228.

Trommolen, M.T.G. The syllable in Dutch, with special reference to diminutive formation. Foris, Dordrecht, 1983.

Weiss, S. and Kulikowski, C. (1991). Computer systems that learn. Morgan Kaufmamn, San Mateo.

Winkel, L.A. Te. Over de verkleinwoorden. Do Taalgids 4: 81-116. 